\title{
ANC-based method for testing converters with random-phase harmonics
}

\author{
V. Kerzérho ${ }^{1}$, F. Azais ${ }^{1}$, M. Comte ${ }^{1}$, P. Cauvet $^{2}$, S. Bernard ${ }^{1}$, and M. Renovell ${ }^{1}$ \\ ${ }^{1}$ LIRMM, University of Montpellier / CNRS - 161 rue Ada, Montpellier, 34392 France \\ v.a.kerzerho@ewi.utwente.nl \\ \{azais, comte, bernard, renovell\}@lirmm.fr \\ ${ }^{2}$ Ophtimalia SAS - 2 Esplanade Anton Philips, Campus Effiscience, Colombelles, 14460 France \\ philippe.cauvet@ophtimalia.com
}

\begin{abstract}
In this paper, we present an extension of the ANC (“Analogue Network of Converters")-based method to characterize the harmonic components of a set of converters with random-phase harmonics using only digital test resources. The ANC-based method was primarily developed under the assumption that the harmonics' phase is proportional to the input phase. This assumption is not valid for all converter architectures, where filtering effects may affect the harmonics' phase. The improved ANC-based method is able to calculate the magnitude of the harmonic components regardless of their phase. The simulation results and the experiments show an excellent agreement between the values measured using the method and the values measured with a usual test setup, for the THD and SFDR parameters.
\end{abstract}

Keywords: converter testing, harmonic characterization, random-phase harmonics

\section{INTRODUCTION}

The democratization of portable data and communication applications like mobile phones, laptops, or audio MP3 players is currently the mainspring of the lookout for integration of many different functions into a single package. Systems-inPackage (SiPs) or Systems-On-Chip (SOCs) that integrate very different analogue or mixed-signal blocks have been developed toward this aim.

Although they offer clear benefits such as device extreme miniaturization or connection length reduction, they imply in compensation very significant test challenges. Indeed in many mixed-signal circuits, the test of the analogue blocks may represent up to $90 \%$ of the whole test effort while these blocks represent only $10 \%$ of the whole chip area. The reason of such a challenge is twofold. Firstly, analogue testing is made of a long sequence of parameter characterization that is performed using very expensive instruments able to accurately measure analogue signals. Secondly, controllability and observability of deeply embedded analogue blocks are much reduced and the possibility of external testing may be limited, as far as the number of pads is greatly reduced. Consequently the internal accessibility of the whole system decreases drastically. Also, as signals become faster and systems are operated at higher speeds, external testing become more susceptible to noise, crosstalk and probing problems.

To overcome these problems, several authors have proposed different Built-In-Self-Test (BIST) techniques where signals are internally generated and/or analyzed [1-7]. Another possible and less expensive solution consists in introducing Design-For-Test (DFT) features to internally transform the analogue signals into digital signals that are made controllable and observable from the chip I/Os [8-11]. As a result, only digital signals are externally handled by non-expensive digital test equipment (Low Cost Tester).

In an example prototype for Set-Top-Box applications, like in most current mixed-signal systems, the converters are among the main components: 2 Analogue-to-Digital Converters (ADC) and 6 Digital-to-Analogue Converters (DAC) are embedded in the same SiP. The today specifications for these converters require a 10-bit resolution, but the next generations will make use of 12-bit converters. Testing this whole set of converters is a very complex task requiring a long test time because of the above mentioned problems of accessibility, signal integrity, accuracy of converter parameter measurements.

In this context, an original DFT technique called “Analogue Network of Converters" (ANC) was developed that permits to test the whole set of embedded ADCs and DACs [12]. An extremely small circuitry is added to the original chip allowing the application of a fully digital lowcost test to the System-in-Package/System-On-Chip. The basic principle consists in setting a test path made of DACs and ADCs. The fault masking is avoided by using a postprocessing algorithm that discriminates harmonics from the different converters inserted in the test path. The ANC-based method can also be exploited to test standalone ADCs using low resolution arbitrary waveform generators as it breaks the rule stating that the test equipment resolution should be at least 2 bits higher than the ADC under test [13].

The ANC-based method was developed with the assumption that the phase of the harmonic components is strictly proportional to the input signal nominal phase. 
However our experiments did not confirm this theoretical assumption. Indeed, we could observe that some harmonics may have a phase that is different from zero, despite the input signal phase is set to zero. In this paper, we present an extension of the primarily developed method to the estimation of the harmonics with a phase which is not correlated to the phase of the input signal.

The paper is organized as follows. Section II gives the fundamental knowledge on data converter testing based on spectral analysis and highlights the issues related to the phase of harmonics. Section III depicts the summarized principle of the ANC-based method. The mathematical developments of the improved ANC-based method are then presented in section IV, followed by validations in section V. Finally section VI concludes the paper.

\section{CONVERTERS, HARMONICS AND PHASES}

A very popular test for data converters relies on spectral analysis. It is usually implemented in a DSP-based test architecture [9, 14]. Basically, a 1-tone sine-wave stimulus is sourced to the converter input, then an FFT analysis is performed on the output sample set. The resulting spectrum is subsequently processed to compute the dynamic parameters such as the Total Harmonic Distortion (THD) or the Spurious Free Dynamic Range (SFDR). The spectrum also permits to estimate an essential static parameter, i.e. the Integral Non Linearity (INL), using the method proposed in [15].

A typical spectrum considering an ADC driven by an ideal sine-wave at frequency $f_{0}$ is illustrated in figure 1 . This spectrum exhibits a fundamental bin at $f_{0}$, the harmonic components being located at multiple values of the fundamental frequency, and noise at other frequencies. The fundamental component corresponds to the input sine-wave while other components are induced by the converter nonidealities.

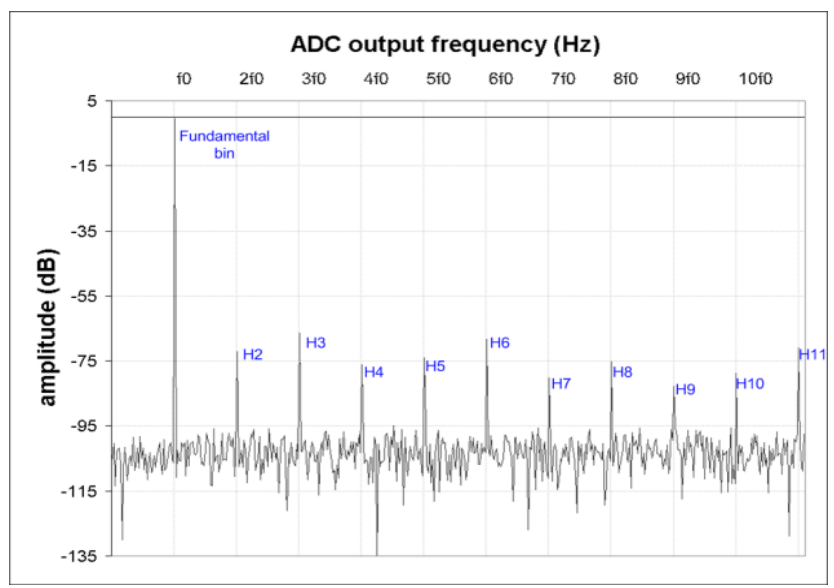

Figure 1. Output spectrum of an ADC under an ideal sine-wave stimulus
From this spectrum, the dynamic parameters are evaluated. In particular, the typical definitions for the THD and SFDR parameters are given by:

$$
\begin{aligned}
& T H D(d B)=10 * \log _{10}\left(\frac{\sum_{k \geq 2} H_{k}^{2}}{F^{2}}\right) \\
& \operatorname{SFDR}(d B)=20 * \log _{10}\left(\frac{F}{\max \left(H_{k}\right)}\right)
\end{aligned}
$$

where $F$ is the amplitude of the fundamental and $H_{k}$ the amplitude of the $\mathrm{k}^{\text {th }}$ harmonic.

To introduce the theoretical fundamentals, let us consider an ideal sine-wave applied to an ADC. Using a Fourier series expansion, the output signal can be expressed by equation (3) given hereafter. This equation distinguishes the sampled sinewave $x(n)$ that would be delivered by an ideal ADC from the sum of all harmonic contributions introduced by the converter non-idealities. Hence $\mathrm{H}_{0}$ corresponds to the offset contribution of the converter and $H_{1}$ corresponds to the contribution of the gain error and non linearity of the converter on the fundamental. The total amplitude of the fundamental component, previously defined as $F$, is the sum of $H_{1}$ and the contribution of the ideal ADC, which is included in $x(n)$ in equation (3):

$$
s(n)=x(n)+\sum_{k \geq 0} H_{k} \cos \left(k\left(\theta_{n}+\theta_{i}\right)+\theta_{k}\right)+\varepsilon(n)
$$

where $n$ is the sample index, $\theta_{i}$ the input phase, $\theta_{k}$ the phase of the $\mathrm{k}^{\text {th }}$ harmonic, $\boldsymbol{d}(\mathrm{)})$ the noise that affect the converted signal, and $\theta_{n}$ the nominal sampling phase given by:

$$
\theta_{n}=2 \pi \frac{M}{N} n
$$

where $N$ is the number of samples and $M$ the number of cycles (i.e. signal periods) in the sample set.

The theoretical developments that mathematically link the harmonic components to the converter INL are given in [15].

The expression of the output signal can be easily linked to the spectrum presented in figure 1 by developing equation (3):

$$
\begin{aligned}
& s(n)=x(n)+ \\
& \sum_{k \geq 0}\left(H_{k} \cos \left(k \theta_{i}+\theta_{k}\right) \cos \left(k \theta_{n}\right)-H_{k} \sin \left(k \theta_{i}+\theta_{k}\right) \sin \left(k \theta_{n}\right)\right)+\varepsilon(n)
\end{aligned}
$$

The sum term corresponds to the Fourier series expansion made of the complex harmonic values resulting from the FFT. Indeed, $H_{k} \cos \left(k \theta_{i}+\theta_{k}\right)$ is equal to the real part of the harmonic computation and $H_{k} \sin \left(k \theta_{i}+\theta_{k}\right)$ is equal to the imaginary part. 
Now if we consider the case of a converter with harmonics' phase proportional to the input phase:

$$
\theta_{k}=k \theta_{i}(\pi)
$$

and by setting the input phase $\theta_{i}$ to zero, equation (5) becomes:

$$
s(n)=x(n)+\sum_{k \geq 0} H_{k} \cos \left(k \theta_{n}\right)+\varepsilon(n)
$$

In this case, the imaginary part of the spectrum is cancelled and only the real part of the spectrum directly corresponds to the amplitude of the spectrum.

However if we consider the case of a converter with harmonics' phase not correlated to the input phase, setting the input phase $\theta_{i}$ to zero leads to:

$$
\begin{aligned}
& s(n)=x(n)+ \\
& \sum_{k \geq 0}\left(H_{k} \cos \left(\theta_{k}\right) \cos \left(k \theta_{n}\right)-H_{k} \sin \left(\theta_{k}\right) \sin \left(k \theta_{n}\right)\right)+\varepsilon(n)
\end{aligned}
$$

In this case, it clearly appears that both the real and the imaginary parts of the spectrum have to be considered and used in the computation of the dynamic parameters.

\section{ANC FUNDAMENTAL PRINCIPLE}

The Analogue Network of Converters, presented in [12], is a DfT concept developed to enable the fully digital test of a set of DACs and ADCs embedded in a complex system. It can also be used to relax the requirements on the test equipment in case of stand-alone converter testing [13].

In the ANC-based method, the analogue outputs of the DACs are basically connected to the analogue inputs of the ADCs, and only digital inputs and outputs are required for test stimulus application and test response analysis. As illustrated in figure 2, the method requires at least two DACs and one ADC. The idea is then to exploit different configurations and different test conditions in order to separate the harmonic contribution of each converter.

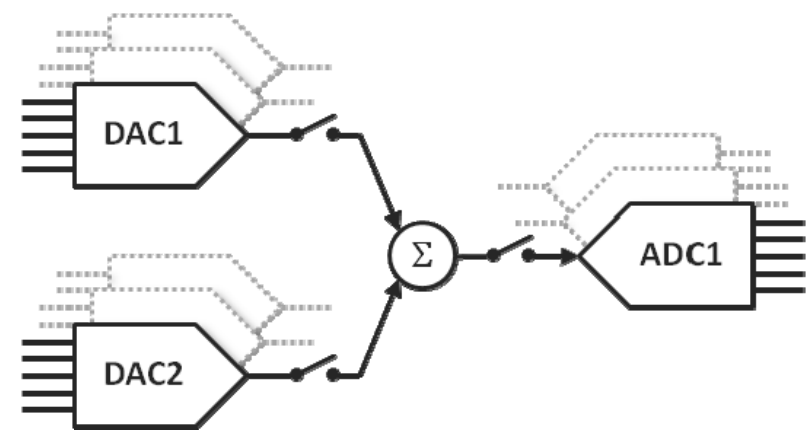

Figure 2. Analog Network of Converters (ANC) concept

The mathematical developments were established considering only static non-idealities of the converters, meaning that the harmonics' phase of each converter was not taken into account. In this case, the model presented in equation (3) simplifies and becomes:

$$
s(n)=x(n)+\sum_{k \geq 0} H_{k} \cos \left(k\left(\theta_{n}+\theta_{i}\right)\right)+\varepsilon(n)
$$

We are therefore in the situation where setting the initial phase $\theta_{i}$ to zero results in the cancellation of the imaginary part of the spectrum, and the real part directly leads to the amplitude of the spectrum.

To illustrate the method, let us first consider a test configuration in which the DAC1 output is directly connected to the ADC1 input. The spectrum of the resulting output signal is computed and the values of the harmonic components $H_{k}{ }^{m}$ are extracted. Obviously, the output signal is affected by errors of both converters. In other words, the resulting spectrum includes the harmonic contribution of DAC1 as well as the harmonic contribution of ADC1. According to equation (9), setting a zero initial phase shift, we can write the following equation:

$$
H_{k}^{m}=\operatorname{Hdac1}_{k}^{F S}+\operatorname{Hadc1}_{k}^{F S}
$$

where $\operatorname{Hdac1}_{k}{ }^{\text {FS }}$ and $\mathrm{Hadc1}_{k}{ }^{\text {FS }}$ correspond to the amplitude of the $\mathrm{k}^{\text {th }}$ harmonic of DAC1 and ADC1 respectively, for a fullscale input signal (FS).

Equation (10) establishes a relation between the harmonic contributions of the two converters involved in the test configuration. In this equation, the left member is known and corresponds to the amplitude of the $\mathrm{k}^{\text {th }}$ spectral bin measured on the spectrum, while the right member corresponds to the unknowns under calculation.

This short example demonstrates the relationship between one configuration and its resulting equation, which leads to the fundamental idea of the ANC-based method. By using different configurations, we can establish different equations. The objective is then to determine the adequate set of configurations that results in a system of independent equations, which can be solved in order to discriminate the harmonic contribution of each converter. We have identified 3 different configurations (DAC1/ADC1, DAC2/ADC1 and DAC1+DAC2/ADC1) associated with 4 different test conditions (related to amplitude and phase of test stimuli) that permit to obtain a system of five independent equations:

$$
\left\{\begin{array}{l}
H_{k}^{m, 1}=\operatorname{Hdac}_{k}^{F S}+\operatorname{Hadc}_{k}^{F S} \\
H_{k}^{m, 2}=\operatorname{Hdac}_{k}^{F S}+\operatorname{Hadc}_{k}^{F S} \\
H_{k}^{m, 3}=\operatorname{Hdac}_{k}^{F S / 2}+\operatorname{Hadc}_{k}^{F S / 2} \\
H_{k}^{m, 4}=\operatorname{Hdac}_{k}^{F S}+\operatorname{Hdac}_{k}^{F S / 2} \cos (k \pi)+\operatorname{Hadc}_{k}^{F S / 2} \\
H_{k}^{m, 5}=\operatorname{Hdac}_{k}^{F S}+\operatorname{Hdac}_{k}^{F S / 2} \cos \left(k \varphi_{1}\right)+\operatorname{Hadc}_{k}^{F S} \cos \left(k \varphi_{2}\right)
\end{array}\right.
$$$$
\text { with } \varphi_{1}=\pi-2 \operatorname{arcos}\left(\frac{1}{4}\right) \quad \text { and } \quad \varphi_{2}=\pi-\operatorname{arcos}\left(\frac{1}{4}\right)
$$ 
Solving this system permits to compute the amplitude of the harmonic components, for each individual converter.

\section{IV.EXTENSION OF THE ANC-BASED METHOD}

A limitation of the ANC-based method described in the previous section is that the phase of the harmonics is not taken into account in the mathematical developments. However, the method was proven efficient in practical experimentations with real converters [16]. This can be explained by the fact that in all experiments, the converters under study presented a phase of harmonics linearly proportional to the input signal nominal phase. In such a situation, when setting the input signal nominal phase to zero, the imaginary part of the spectrum is cancelled and the real part directly gives the amplitude of the spectrum. The system of equations established from the model given by equation (9) is therefore valid and leads to a correct estimation of the harmonic contribution of each individual converter. However the assumption that the harmonics' phase is linearly proportional to the input signal nominal phase is not always verified and highly depends on the converter architecture. We have seen that this assumption is often verified for types of architecture that use a sample and hold stage, but it cannot be assessed for other architectures where filtering and noise may affect the harmonics' phase. Our objective is consequently to improve the ANC-based method so that it can cover all the real-life cases.

The fundamental principle of this improved version of the ANC-based method is the same, but the mathematical developments have now to be established using the complete model valid in the general case, i.e. the model described by equation (3).

So let us consider again the configuration in which the DAC1 output is directly connected to the ADC1 input. Obviously, the output signal is still affected by errors of both converters. Now, considering the general case described in equation (3) and setting the input signal nominal phase $\theta_{i}$ to zero, we can write the following equations:

$$
\left\{\begin{array}{l}
\operatorname{Re}\left(H_{k}^{m}\right)=\operatorname{Hdac1}_{k}^{F S} \cos \left(\theta_{\text {dac1,k}}^{F S}\right)+\operatorname{Hadc}_{k}{ }_{k}^{F S} \cos \left(\theta_{\text {adc1,k }}^{F S}\right) \\
\operatorname{Im}\left(H_{k}^{m}\right)=-H d a c 1_{k}^{F S} \sin \left(\theta_{\text {dac1,k }}^{F S}\right)-H a d c 1_{k}^{F S} \sin \left(\theta_{\text {adc1 }, k}^{F S}\right)
\end{array}\right.
$$

Similarly, we can derive equations for all configurations and test conditions involved in the ANC-based method. We finally obtain a system of 10 independent equations given hereafter.

This system can be solved to determine the real and imaginary parts of harmonic contribution related to the different converters. From this, the dynamic parameters such as THD and SFDR can be computed for each converter. Solving this system of equations therefore makes the independent characterization of each converter possible.

$$
\begin{aligned}
& \int \operatorname{Re}\left(H_{k}^{m, 1}\right)=H d a c 1_{k}^{F S} \cos \left(\theta_{d a c 1, k}^{F S}\right)+H a d c 1_{k}^{F S} \cos \left(\theta_{\text {adc1,k }}^{F S}\right) \\
& \operatorname{Im}\left(H_{k}^{m, 1}\right)=-H d a c 1_{k}^{F S} \sin \left(\theta_{\text {dac1, }}^{F S}\right)-H a d c 1_{k}^{F S} \sin \left(\theta_{\text {adc } 1, k}^{F S}\right) \\
& \operatorname{Re}\left(H_{k}^{m, 2}\right)=H d a c 2_{k}^{F S} \cos \left(\theta_{\text {dac } 2, k}^{F S}\right)+H a d c 1_{k}^{F S} \cos \left(\theta_{\text {adc } 1, k}^{F S}\right) \\
& \operatorname{Im}\left(H_{k}^{m, 2}\right)=-H d a c 2_{k}^{F S} \sin \left(\theta_{d a c 2, k}^{F S}\right)-\operatorname{Hadc}_{k}^{F S} \sin \left(\theta_{\text {adc1, }}^{F S}\right) \\
& \operatorname{Re}\left(H_{k}^{m, 3}\right)=H d a c 2_{k}^{F S / 2} \cos \left(\theta_{\text {dac } 2, k}^{F / 2}\right)+H a d c 1_{k}^{F S / 2} \cos \left(\theta_{\text {adc1 }, k}^{F S}\right) \\
& \operatorname{Im}\left(H_{k}^{m, 3}\right)=-H d a c 2_{k}^{F S / 2} \sin \left(\theta_{\text {dac } 2, k}^{F S / 2}\right)-H a d c 1_{k}^{F S / 2} \sin \left(\theta_{\text {adc } 1, k}^{F S / 2}\right) \\
& \operatorname{Re}\left(H_{k}^{m, 4}\right)=\left[\begin{array}{l}
\operatorname{Hdac1}_{k}^{F S} \cos \left(\theta_{\text {dac } 1, k}^{F S}\right)+ \\
\operatorname{Hdac}_{k}^{F S / 2} \cos (k \pi) \cos \left(\theta_{\text {dac } 2, k}^{F S / 2}\right)+ \\
\operatorname{Hadc1}_{k}^{F S / 2} \cos \left(\theta_{\text {adc } 1, k}^{F S / 2}\right)
\end{array}\right] \\
& \operatorname{Im}\left(H_{k}^{m, 4}\right)=-\left[\begin{array}{l}
\operatorname{Hdac1}_{k}^{F S} \sin \left(\theta_{\text {dac1,k }}^{F S}\right)+ \\
\operatorname{Hdac2}_{k}^{F S / 2} \cos (k \pi) \sin \left(\theta_{\text {dac } 2, k}^{F S / 2}\right)+ \\
\operatorname{Hadc1}_{k}^{F S / 2} \sin \left(\theta_{\text {adc1, }}^{F S}\right)
\end{array}\right] \\
& \operatorname{Re}\left(H_{k}^{m, 5}\right)=\left[\begin{array}{l}
\operatorname{Hdac1}_{k}^{F S} \cos \left(\theta_{\text {dac1, }}^{F S}\right)+ \\
\operatorname{Hdac2}_{k}^{F S / 2}\left[\cos \left(k \varphi_{1}\right) \cos \left(\theta_{\text {dac } 2, k}^{F S / 2}\right)-\sin \left(k \varphi_{1}\right) \sin \left(\theta_{\text {dac } 2, k}^{F S / 2}\right)\right] \\
\operatorname{Hadc1}_{k}^{F S}\left[\cos \left(k \varphi_{2}\right) \cos \left(\theta_{\text {adc1, }}^{F S}\right)-\sin \left(k \varphi_{2}\right) \sin \left(\theta_{\text {adcl }, \mathrm{k}}^{F S}\right)\right]
\end{array}\right]+ \\
& \operatorname{Im}\left(H_{k}^{m, 5}\right)=-\left[\begin{array}{l}
\operatorname{Hdac1}_{k}^{F S} \sin \left(\theta_{\text {dac1,k }}^{F S}\right)+ \\
\operatorname{Hdac}_{k}^{F S / 2}\left[\sin \left(k \varphi_{1}\right) \cos \left(\theta_{\text {dac } 2, k}^{F S / 2}\right)+\cos \left(k \varphi_{1}\right) \sin \left(\theta_{\text {dac } 2, k}^{F S / 2}\right)\right]+ \\
\operatorname{Hadc1}_{k}^{F S}\left[\sin \left(k \varphi_{2}\right) \cos \left(\theta_{\text {adc1, }}^{F S}\right)+\cos \left(k \varphi_{2}\right) \sin \left(\theta_{\text {adc1 }, k}^{F S}\right)\right]
\end{array}\right] \\
& \text { with } \quad \varphi_{1}=\pi-2 \operatorname{arcos}\left(\frac{1}{4}\right) \quad \text { and } \quad \varphi 2=\pi-\operatorname{arcos}\left(\frac{1}{4}\right)
\end{aligned}
$$

\section{VALIDATION}

The theoretical extension of the ANC-based test method has been validated through simulation, using the converter model described in [12]. Converter non-idealities are included in the model based on real-life INL values extracted from measurements on several data converters. Sampling jitter and thermal noise are also considered. In addition, we also include a possible injection of an arbitrary phase shift on the converter harmonics.

In order to validate the improved ANC-based method, we have considered a set of three converters, i.e. two DACs and one ADC. First, we have simulated each data converter in a stand-alone configuration and we have applied a usual test to get reference values for the THD and SFDR parameters. Then, we have simulated the set of converters in the different configurations and test conditions defined in the ANC-based method. From these five acquisitions, we have established the system of independent equations that links the measured harmonic contribution to the individual harmonic contribution of each converter (considering only the real part of the spectrum for the original ANC-based method and both the real and imaginary parts of the spectrum for the improved ANCbased method) and we have solved the system. Finally, we have computed the THD and SFDR parameters using the estimated amplitude of the harmonic components.

Results are summarized in Table I and II, which compare the values of the THD and SFDR parameters computed using 
the stand-alone configuration to the values of the THD and SFDR parameters computed using either the original or the improved ANC-based method, with and without phase shift injected on the converters harmonics.

TABLE I. MEASUREMENT OF THD AND SFDR VALUES WITHOUT PHASE SHIFT ON HARMONICS' PHASE

\begin{tabular}{cccc|ccc} 
& \multicolumn{3}{c|}{ THD (dB) } & \multicolumn{3}{c}{ SFDR (dB) } \\
& DAC1 & DAC2 & ADC & DAC1 & DAC2 & ADC \\
\hline Ref & -77.9 & -74.3 & -66.1 & 78.9 & 76.1 & 70.5 \\
$\begin{array}{c}\text { Original } \\
\text { ANC }\end{array}$ & -77.2 & -74.1 & -65.8 & 79.3 & 75.6 & 69.8 \\
$\begin{array}{c}\text { Inproved } \\
\text { ANC }\end{array}$ & -77.3 & -74.2 & -65.7 & 79.3 & 75.6 & 69.5 \\
& & & & & &
\end{tabular}

TABLE II. MEASUREMENT OF THD AND SFDR VALUES WITH PHASE SHIFT INJECTED ON HARMONICS’ PHASE

\begin{tabular}{cccc|ccc} 
& \multicolumn{3}{c|}{ THD (dB) } & \multicolumn{3}{c}{ SFDR (dB) } \\
& DAC1 & DAC2 & ADC & DAC1 & DAC2 & ADC \\
\hline Ref & -77.9 & -74.4 & -66.1 & 78.9 & 76.1 & 70.2 \\
$\begin{array}{c}\text { Original } \\
\text { ANC }\end{array}$ & -73.6 & -73.2 & -67.0 & 76.8 & 76.6 & 68.5 \\
$\begin{array}{c}\text { Inproved } \\
\text { ANC }\end{array}$ & -77.7 & -74.4 & -66.2 & 78.8 & 76.1 & 70.4
\end{tabular}

Analyzing these results, it can be seen that when the converters harmonics are not affected by a random phase shift, both the original and the improved versions of the ANC-based method produce an accurate measurement of the THD and SFDR parameters, with an estimation error that remains below $0.7 \mathrm{~dB}$. However when the converters' harmonics are affected by a random phase shift, the performances of the original ANC-based method degrades with an estimation error that increases up to $4.3 \mathrm{~dB}$ for the THD parameter of DAC1. In contrast, the improved ANC-based method gives very accurate measurements, with an estimation error lower than $0.2 \mathrm{~dB}$. These results clearly validate the efficiency of the improved ANC-based method.

\section{VI.CONCLUSION}

In this paper, we have presented an extension of the ANCbased method in order to handle converters with random-phase harmonics. Indeed, the ANC-based method was originally developed under the assumption that the harmonics' phase is proportional to the input phase. Although this assumption is often verified, it is not valid for all converter architectures. New mathematical developments based on a general model that includes the harmonics' phase of converters were therefore established. The efficiency of the improved ANCbased test was evaluated through simulations, demonstrating an excellent agreement between the THD and SFDR values measured with a classical test setup and the THD and SFDR values computed with the method.
Compared to the initial ANC-based test method, no additional resources are required, and the number of test configurations and test acquisitions remains the same. Only the size of the independent equation system is different, growing from 5 to 10 . However, it is worth noting that this computation is performed off-chip using the tester digital signal processing resources and has a negligible impact on the cost of the test procedure. These developments therefore offer an efficient generic method to perform the harmonic characterization of a set of data converters using only digital external test equipment, without any assumption on the converter architecture and potential phase shift of the converter harmonics.

\section{REFERENCES}

[1] M. Toner, G. Roberts, "A BIST scheme for an SNR test of a sigmadelta ADC”, Proc. International Test Conference, pp.805 - 814, 1993.

[2] M. Toner, G. Roberts, “A BIST Technique for a Frequency Response and Intermodulation Distortion Test of a Sigma-Delta ADC”, Proc. IEEE VLSI Test Symposium, pp.60-65, 1994.

[3] M.J. Ohletz, "Hybrid Built In Self Test (HBIST) for Mixed Analog/Digital Integrated Circuits”, Proc. IEEE European Test Conference, pp.307-16, 1991.

[4] S.K. Sunter, N. Nagi, "A simplified polynomial-fitting algorithm for DAC and ADC BIST”, Proc. IEEE International Test Conference, pp.389-395, 1997.

[5] F. Azais, S. Bernard, Y. Betrand, M. Renovell, "Towards an ADC BIST scheme using the histogram test technique”, Proc. IEEE European Test Workshop, pp.53 - 58, 2000.

[6] F. Azais, S. Bernard, Y. Bertrand, M. Renovell, "Implementation of a linear histogram BIST for ADCs”, Proc. Conference and Exhibition Design, Automation and Test in Europe, pp.590 - 595, 2001.

[7] K. Arabi, B. Kaminska, J. Rzeszut, “A New Built-In Self Test Approach For Digital-to-Analog and Analog-to-Digital Converters”, Proc. IEEE International Conference on Computer-Aided Design, pp.491-494, 1994.

[8] N. Nagi, A. Chatterjee, J. Abraham, "A Signature Analyzer for Analog and Mixed-Signal Circuits”, Proc. IEEE International Conference on Computer Design, pp.284-287, 1994.

[9] M. Mahoney, "DSP-Based Testing of Analog and Mixed-Signal Circuits”, IEEE Computer Society Press, ISBN 0-8186-0785-8, 1987.

[10] M. Burns, G.W. Roberts, “An Introduction to mixed-signal IC test and measurement”, Oxford university press, ISBN 0195140168, 2001.

[11] J.L. Huertas, "Test and design-for-testability in mixed-signal integrated circuits”, Springer Press, ISBN 14020077246, 2004.

[12] V. Kerzérho, P. Cauvet, S. Bernard, F. Azaïs, M. Comte, M. Renovell ““Analogue Network of Converters”: a DFT Technique to Test a Complete Set of ADCs and DACs Embedded in a Complex SiP or SOC”, Proc. IEEE European Test Symposium, pp. 159-164, 2006.

[13] V. Kerzérho, P. Cauvet, S. Bernard, F. Azaïs, M. Renovell, M. Comte, O. Chakib, “ADC Production Test Technique Using LowResolution Arbitrary Waveform Generator”, VLSI Design, vol. 2008, Article ID 482159, 8 pages, 2008.

[14] "IEEE standard for terminology and test methods for analog-to-digital converters”, IEEE Std 1241-2000.

[15] J.M. Janik, V. Fresnaud, “A Spectral approach to estimate the INL of A/D converter”, Computer Standards \& Interfaces journal, Vol. 29(1), pp. $31-37,2007$.

[16] V. Kerzérho, P. Cauvet, S. Bernard, F. Azaïs, M. Renovell, M. Comte "A novel DFT technique to test a complete set of ADC's and DAC's embedded in a complex SiP”, IEEE Design and Test of Computers journal, May-June 2006, Vol. 23, Issue 3, pp 234-243. 